\title{
A Comparison of Probabilistic and Deterministic Analysis for Human Space Exploration
}

\author{
R. Gabe Merrill ${ }^{1}$ and Mark Andraschko ${ }^{2}$ \\ Analytical Mechanics Associates Inc, Hampton, Virginia, 23666 \\ Chel Stromgren ${ }^{3}$ \\ Science Applications International Corporation, Mclean, Virginia, 22102 \\ and \\ Bill Cirillo ${ }^{4}$, Kevin Earle ${ }^{5}$ and Kandyce Goodliff ${ }^{6}$ \\ NASA Langley Research Center, Hampton, Virginia, 23681
}

\begin{abstract}
Human space exploration is by its very nature an uncertain endeavor. Vehicle reliability, technology development risk, budgetary uncertainty, and launch uncertainty all contribute to stochasticity in an exploration scenario. However, traditional strategic analysis has been done in a deterministic manner, analyzing and optimizing the performance of a series of planned missions. History has shown that exploration scenarios rarely follow such a planned schedule. This paper describes a methodology to integrate deterministic and probabilistic analysis of scenarios in support of human space exploration. Probabilistic strategic analysis is used to simulate "possible" scenario outcomes, based upon the likelihood of occurrence of certain events and a set of pre-determined contingency rules. The results of the probabilistic analysis are compared to the nominal results from the deterministic analysis to evaluate the robustness of the scenario to adverse events and to test and optimize contingency planning.
\end{abstract}

\section{Introduction}

Cenario options for NASA's planned return to the Moon and continued exploration over the next several decades $\checkmark$ are being strategically evaluated through integrated probabilistic and deterministic analyses. Decision-making is supported through the development of key performance metrics that encompass both the planned and expected value of evaluated exploration scenarios. The purpose of strategic analysis is to provide decision-makers with an improved understanding of the performance, cost, risk, schedule, and value implications of their decisions ${ }^{1}$. It is not intended as a design process, but as an analytical decision support process to enable informed decision-making. Analysis models support these activities by enabling a more thorough exploration of the trade space and providing performance results that serve as the basis for affordability, technology assessment, and expected value analyses.

Traditional deterministic analysis allows for an evaluation of the nominal performance of space missions. While this is a critical component in the development of an exploration scenario, using this approach alone neglects uncertainty associated with human space exploration. Vehicle reliability, technology development risk, budgetary uncertainty, and launch uncertainty all contribute to stochasticity in an exploration scenario. Strategic analysis that allows for both deterministic and probabilistic modeling provides a better understanding of the system's range of behaviors due to various modeled uncertainties.

This paper describes an approach used by the NASA Constellation Program Strategic Analysis Team (SAT) to integrate deterministic and probabilistic analysis of human lunar exploration scenario options. Specifically, the paper

${ }^{1}$ Project Engineer, 303 Butler Farm Rd, Suite 104A, AIAA Member.

${ }^{2}$ Project Engineer, 303 Butler Farm Rd, Suite 104A, AIAA Member.

${ }^{3}$ Chief Scientist, Modeling and Analysis Division, 1710 SAIC Drive, AIAA Member.

${ }^{4}$ Senior Researcher, Space Missions Analysis Branch, MS 462, AIAA Member.

${ }^{5}$ Aerospace Engineer, Space Missions Analysis Branch, MS 462, AIAA Member.

${ }^{6}$ Aerospace Engineer, Space Missions Analysis Branch, MS 462, Senior AIAA Member. 
will describe the differences between deterministic and probabilistic analysis, the processes used to evaluate each, and the types of calculations that are generated using both types of analysis. Finally, the paper will describe the types of decisions that can be supported by each type of analysis and the overall value of performing an integrated assessment.

\section{Integrated Analysis}

A robust scenario analysis capability is comprised of both deterministic and probabilistic analysis capabilities and takes advantage of the synergies that exist between the two types of analysis. The methodology used by the SAT to support the analysis of exploration scenarios uses both types of analysis in a progressive manner, designed to minimize computational effort while allowing a thorough examination of the scenario trade space. Figure 1 illustrates the integrated relationship between the deterministic and probabilistic analyses conducted.

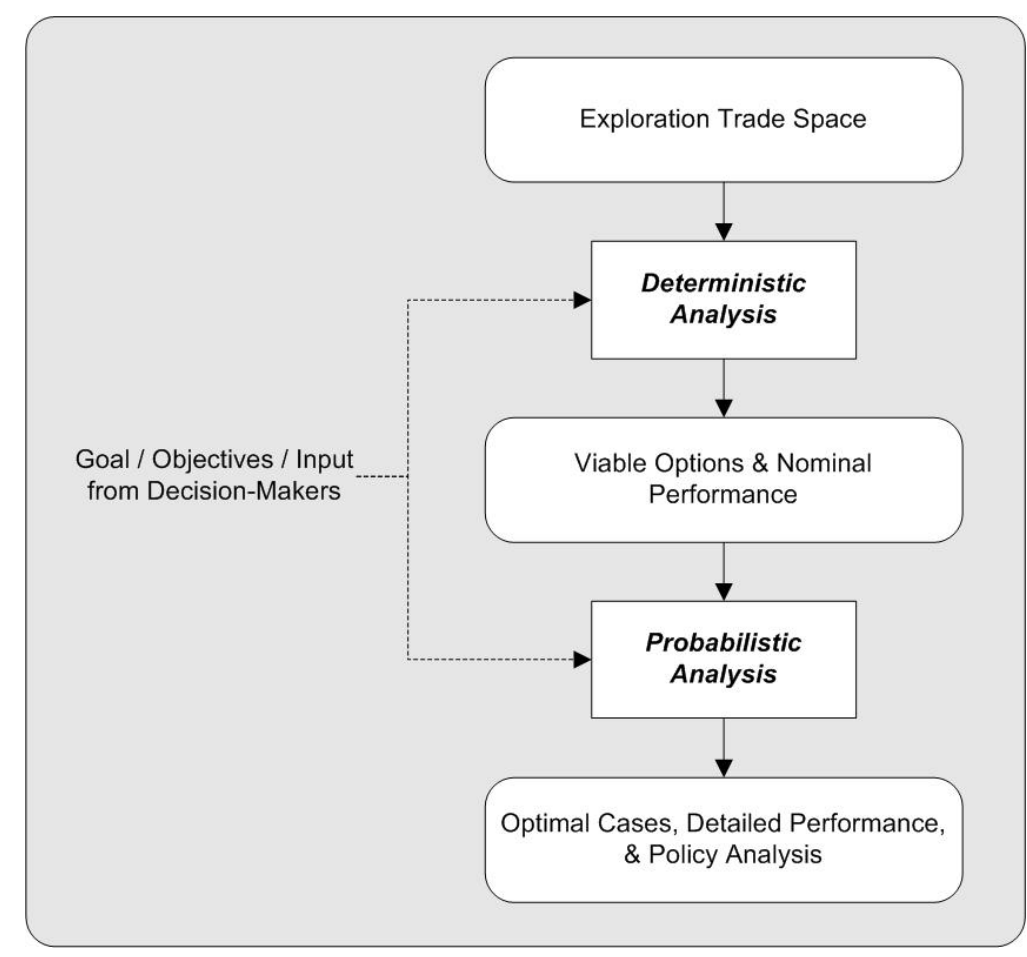

Figure 1. Integrated Strategic Analysis.

Deterministic analysis is used to evaluate a broad scenario trade space and narrow down the set of options to those that are viable, given performance, cost, and safety constraints. Because deterministic analysis requires relatively low computational time, it is possible to rapidly iterate through scenario analysis at this level of low fidelity. Satisfaction of requirements through an iterative analysis cycle can be quickly completed, allowing a large number of candidate scenarios to be rapidly developed and evaluated. In addition, the quick turn-around of deterministic analysis provides for sensitivity analysis of key scenario parameters. Specific issues that are evaluated at the deterministic level include:

- Mission requirements

- Mission capabilities

- Flight loading

- Element phasing

- Power requirement, generation, and storage

- Crew time

- Nominal productivity

- Cost

- Mission safety \& reliability 
Probabilistic analysis allows for more thorough examination of scenario options that are found to be deterministically viable. Analysis is expanded to include issues such as the robustness of scenarios to mission failures and programmatic delays, the effectiveness of contingency planning, and the expected productivity given the uncertainty in the system. The results from the analysis then allow decision-makers to further select and refine scenario options to maximize value and ensure long-term robustness and sustainability. Specific issues that are evaluated at the probabilistic level include:

- Expected failures

- Flight loading

- Expected productivity

- Expected cost

- Contingency planning

- Mission safety \& reliability

The integrated use of both methodologies allows the SAT to progressively increase the sophistication of analysis as the number of candidate scenario options is narrowed. This approach enables full deterministic exploration of the available scenario trade space at a high level and then full, robust probabilistic evaluation of options that appear viable at the deterministic level.

\section{Deterministic Analysis}

The deterministic analysis tools developed and employed by the SAT enables evaluation of several metrics or figures of merit (FOMs) describing scenario performance. The first step in deterministic analysis is scenario definition, which serves as the foundation for more detailed affordability, risk, crew time, and value assessments.

\section{A. Scenario Definition}

Definition of a human space exploration scenario is an iterative process of planning and analysis which structures a complete set of missions that nominally meet scenario requirements; the basic phasing and structure of the scenario being driven by scenario objectives and expectations specified by study leadership. The deterministic model relies on two basic types of input. Specific technical performance data for elements and transportation options, developed by design teams and subject matter experts, are imported into the model and form the basis of a scenario. To develop a specific scenario option, users then input specific information regarding how each mission in the scenario is structured. This information includes: nominal mission date, number of crew, length of crewed surface duration, delivery capacity of the transportation system, and payloads (vehicles and surface elements) delivered. Once the initial scenario structure has been defined, the logistics necessary to sustain crew and element operations are calculated and loaded into carriers for delivery prior to their date of use. If all requirements cannot be met, the scenario is modified and the calculation repeated until the scenario is closed. Scenario closure is achieved when the elements, logistics and carriers can be manifested within the available capacity of the transportation system to support the crew surface stay duration for each mission.

Exploration scenarios are modeled discretely, which allows element properties to be modeled with fixed / constant rate values, and reduces the quantity of required inputs. This modeling approach enables the flexibility needed for thorough interrogation of any region of a large, relatively unconstrained trade space. Sensitivity analysis and trade studies provide additional insight into the behavior of the nominal scenario by varying key system parameters, such as the physical characteristics of the elements, their associated logistics, required crew consumables, and the logistics packaging methodology.

The technical performance data regarding transportation and surface element options are stored in an element library linked to the deterministic tool set. Prior to scenario manifesting, groups of engineers or subject matter experts develop the set of elements based on direction from study leadership. These elements can be categorized into general capability categories with an element design team for each. The main element disciplines are habitation and crew support, power, mobility, element handling, communication, and in-situ resource utilization (ISRU) with additional cross-cutting systems engineering teams that support interaction among element design teams. The element conceptual designs are then leveraged for development of a human lunar exploration scenario through a deterministic analysis closure process that is graphically represented in Figure 2. 


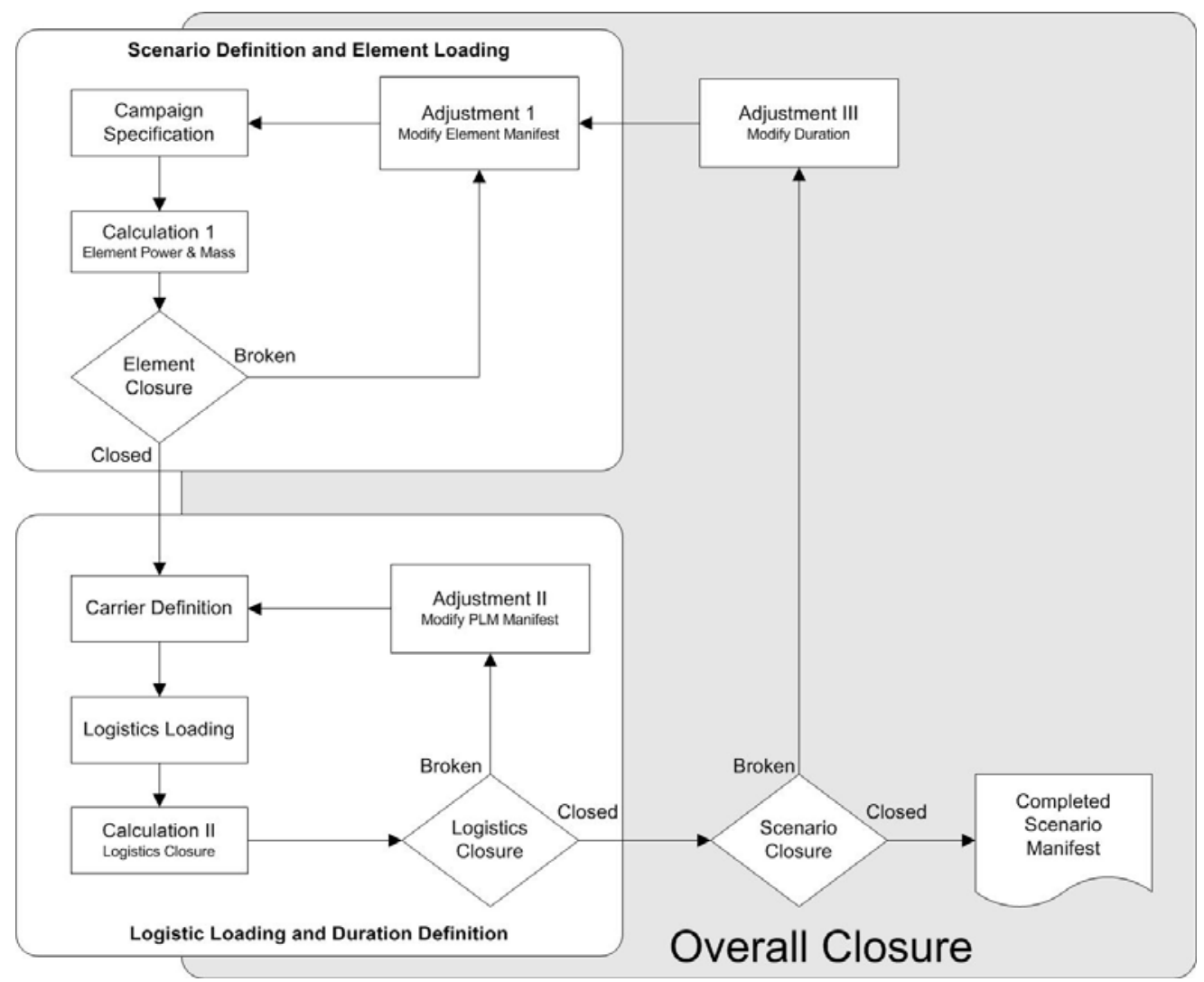

Figure 2. Deterministic Analysis Closure Process.

Top-level objectives and assumptions provided by study leadership drive the definition of a scenario using applicable elements and other assumptions that have been captured within the analysis model. Constructing a manifest involves the following activities:

1) Mission specification and element loading

2) Logistics loading and duration definition

3) Establishing closure

The first step required to define a lunar exploration scenario is the development of a basic specification for each mission including the type of mission and the planned date of the mission. The basis for this specification is the annual flight rate to the Moon and the timing of those flights. Initial timing is dictated by the desired Human Lunar Return (HLR) or "Boots on the Moon" date with scheduling of the remainder of the scenario dictated by the planned flight rate profile, ground infrastructure turnaround capabilities, and lighting conditions at the target location on the lunar surface at the time of landing and throughout the crewed mission durations. The planned flight rate establishes the number of flights to be planned for each fiscal year. The turnaround capabilities establish the minimum amount of time between missions. These are key drivers of both the timing of the planned flights and the speed at which infrastructure can be built up on the Moon.

Once the initial flight schedule is defined, specific surface elements are loaded onto the missions for delivery based on goals and objectives set forth by study leadership for a given scenario. In scenarios that require extended or continuous human presence on the Moon, elements are loaded on missions in such a way that the infrastructure on the surface is built up into an "Outpost" that enables long term goals while also satisfying upfront requirements. Elements must be delivered sequentially such that system resources and requirements are balanced or "closed". There must be enough power generation and storage capability provided by the power elements to enable the habitation elements to keep the crew alive while enabling use of additional elements for all required crew operations such as outpost infrastructure assembly, lunar exploration and characterization, and ISRU during the planned mission timeframe.

After mission and element loading specification, the next step in the scenario definition is calculation of logistics requirements and the loading of those logistics onto missions ${ }^{2}$. Logistics and crew consumable requirements are 
determined based upon the elements at that location, the number of crew and their surface duration, as well as a specification of the ECLSS system, ISRU production, and resource stockpile availability. Spares and maintenance requirements are determined based upon the inventory of elements that have been delivered to the surface. Utilization requirements, specifically science requirements, are determined based on scenario objectives and the flight schedule.

Pressurized carriers, tanks for gasses and liquids, and palletized carriers for unpressurized goods are loaded onto individual missions. The required logistics are then loaded onto each mission within specific carriers for delivery prior to the date that they are required for use. Any cases in which the logistics can not be loaded prior to need date due to limited capacity are flagged. For these cases, the logistics are overloaded onto those missions and packaging factors are used for the carriers. This labels a mission that is unable to deliver the required cargo and defines the additional cargo capacity required to close the mission.

The crewed duration of each mission is then iterated and the analysis process repeated until the crewed duration of each mission can be supported by both the elements available and the logistics that are carried prior to the mission. At this point, the scenario has been defined and can be used for further deterministic analysis, sensitivity analysis, or passed to the probabilistic analysis model.

\section{B. Risk Analysis}

The deterministic analysis includes an estimation of scenario risk, including both safety and uncertainty. On a mission-by-mission basis, the risk evaluation model has the capability to estimate the probability of different possible mission end states. The risk evaluation model utilizes vehicle reliability to determine the overall likelihood of failure, vehicle maturity to account for the experience and growth accumulated from the prior missions, and evaluation of launch probabilities in multi-launch scenarios to incorporate vehicle orbital lifetimes and historical launch probability data. Certain results from the risk model are reported out as independent FOMs, including the probability of loss of crew and the probability of loss of key surface architecture elements. The remaining risk results are used to drive the probabilistic analysis of the scenario definition. For more information on the Risk Analysis, please refer to reference 1 .

\section{Affordability}

Affordability analysis incorporates both the integration and manipulation of various cost estimates into a holistic model for scenario cost prediction. In order for analysis to include all germane affordability considerations, the entire life cycle cost (LCC) of various lunar exploration elements is required (with the sole exception of disposal costs since end of scenarios may be open-ended). Affordability analysis modeling is handled both deterministically and probabilistically in order to enable rapid trade space analyses and uncertainty quantification for certain cases. Deterministic analysis focuses on integrating the data together to enable the addition/removal of lunar exploration elements, easy modification of element scenario definition dates, and manipulation of other driving assumptions to allow full trade space exploration. The deterministic results (i.e., point estimates) provide tremendous insight into the behavior of candidate lunar exploration scenarios and their contributing costs. For more information on Affordability, please refer to reference 1 .

\section{Crew Time Analysis}

The deterministic analysis capability includes a crew time model, which evaluates, at a high-level, the availability of crew surface time including both time for required activities, and time available for utilization. The model also specifically evaluates the portion of crew time available for intra-vehicular activities (IVA) and extravehicular activities (EVA). Analysis of crew time availability is based on the scenario description for which aggregate time availability and requirements over the entire duration of each crewed surface increment is modeled. The process for evaluating crew time involves a series of calculation steps:

- Total crew work time available in the increment

- Crew time required for basic required tasks

- IVA time required for logistics and maintenance

- $\quad$ EVA time required for logistics and maintenance

- Total possible EVA time

- Crew time available for IVA utilization

- Crew time available for EVA utilization

The total amount of crew time available in each increment represents the surface time when the crew is expected to do productive work. This available time is based on the total number of surface days, a standard workweek 
schedule, and a standard workday schedule. Work time does not include basic activities such as eating, recreation, or resting. Once the total amount of time has been determined, the amount of crew time that is taken up by routine activities is calculated. Generally, these activities are calculated as a weekly requirement and applied against the entire increment duration. Routine activities include: training, crew tag-ups, medical evaluations, public relations, inventory, and logistics transfer. In addition, crew time requirements for maintenance and repair are also calculated. Maintenance and repair time is calculated based on the inventory of goods that have been delivered to the surface and predicted annual maintenance time requirements in crewed and dormant modes. Maintenance and repair time is divided into two types: IVA time, which takes place within the habitat, and EVA time, which occurs out of the habitat.

The number of total possible EVAs that can be completed by the crew is then calculated based upon a nominal weekly EVA schedule and the total increment duration. The total number of EVAs includes those required for logistics and maintenance and those available for utilization. For the purposes of this analysis, it is assumed that the crew will complete all EVA opportunities. The requirements for routine activities and IVA maintenance and repair, as well as the total time that the crew will spend on EVA, are then subtracted from the total available work time to determine the total time that is available for IVA utilization. This is the time that the crew has available while in the habitat to conduct science and other utilization activities. Similarly, the amount of EVA time required for repair and maintenance is subtracted from the total available EVA time. The remainder represents the amount of time while on EVA that the crew has to conduct science and other utilization activities.

The crew time model does allow a certain fraction of required maintenance and repair time to be deferred to later crewed missions. If, on any particular mission, the amount of time required to complete maintenance and repair cannot be satisfied, the deficit is added to the maintenance and repair requirement for the following mission. If the deficit exceeds a pre-defined limit on any given mission, a flag is raised in the results indicating that there may be a gap in availability.

\section{E. Use of Deterministic Results}

The results produced through deterministic analysis allow for selective filtering of scenario options. Initially, from the broad trade space of possible scenarios, it is possible to select those groups of scenarios that are viable under the constraints and goals specified by project management. Specific areas of analysis that might lead to scenarios being deemed unviable include:

- Lack of transportation capacity to deliver specified elements

- Lack of transportation capacity to deliver required logistics

- Failure in outpost supportability due to element phasing

- Lack of crew time to complete required activities

- Failure to meet programmatic goals for key dates

- Failure to adequately satisfy scenario objectives

- Failure to meet budgetary constraints

Scenarios that fail due to any of these reasons can rapidly be removed from the trade space. The deterministic tools can then be used to further refine the trade space through direct comparison of the nominal FOMs for various options. Scenarios which are clearly inferior across a broad set of FOMs do not warrant further analysis. In this manner, the trade space can be reduced to those scenario options that offer clearly superior performance against one or more of the FOMs. This reduced set of candidate scenarios are those to which the more sophisticated, computationally intense, probabilistic analysis is applied.

\section{Probabilistic Analysis}

The SAT has developed a methodology and associated models that execute probabilistic analysis of lunar exploration scenarios. These probabilistic tools are used to simulate the real-world outcome of lunar exploration scenarios, based upon the probability of occurrence for non-nominal events, the expected consequence and delays associated with those events, and established contingency operations polices. Using this data, a large number of possible scenarios are simulated, each a possible instantiation of the actual scenario. The details of the probabilistic analysis process are shown in Figure 3. 


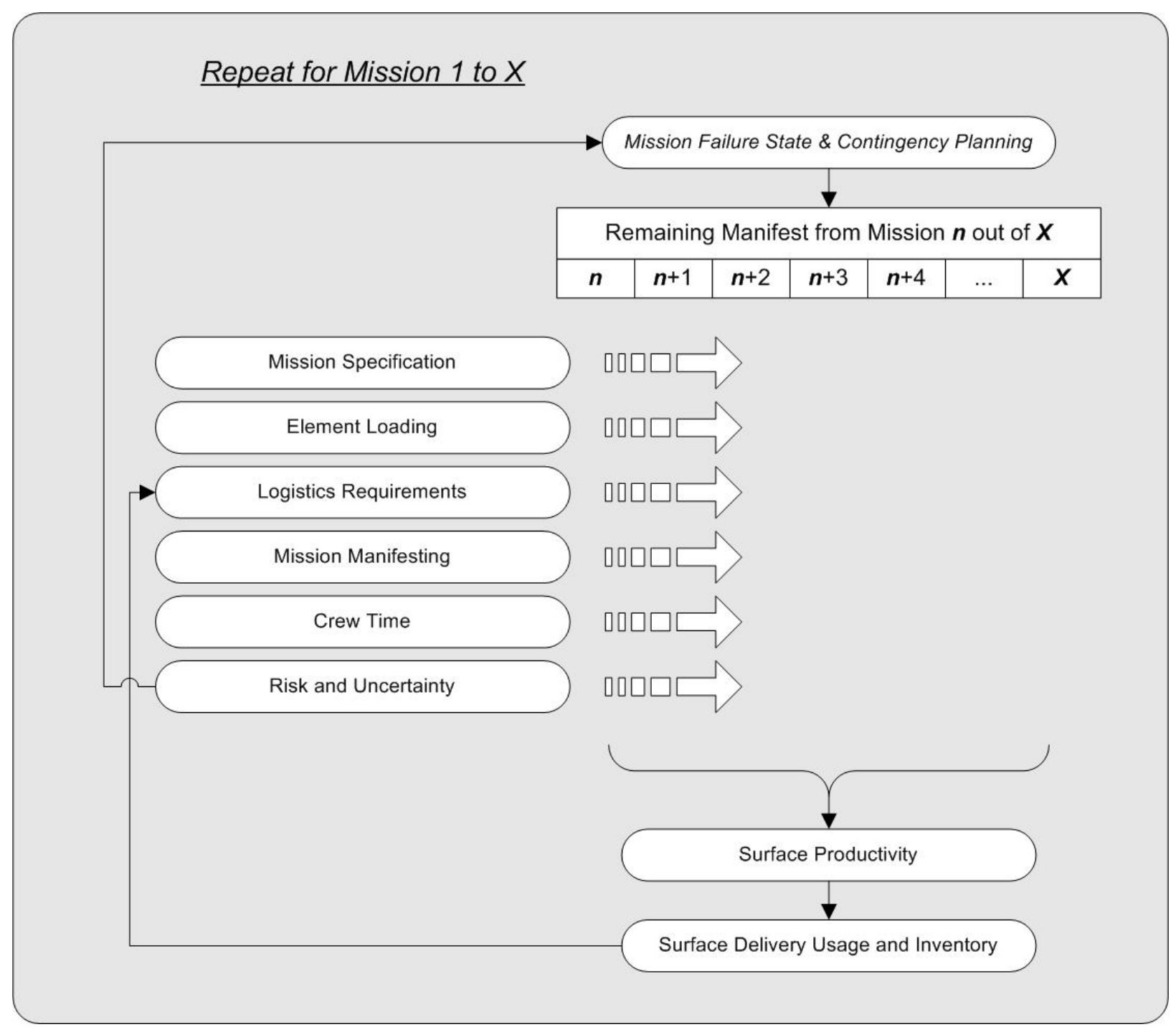

Figure 3. Probabilistic Analysis Process.

\section{A. Generation of Probabilistic Cases}

The probabilistic analysis involves generating a large number of possible real-world outcomes for a given exploration scenario. These real-world outcomes reflect the probability that adverse events can occur and include the consequence of those events. Because each individual case that is evaluated reflects only a single possible outcome, it is necessary to run a large number of cases in order to span the total range of possible outcomes. The probabilistic model is simply an engine that generates the probabilistic cases and collects the results. The actual analysis of independent cases is accomplished through a manipulation of the deterministic tools described above.

Within each simulated probabilistic case, the engine controls a mission-by-mission temporal simulation. Leveraging the deterministic analysis methodology, the probabilistic engine evaluates the success or failure of the current mission. The outcome of the current mission is simulated based on probability distributions for all possible non-nominal events using a mission event tree. The probability for failure events, calculated for the current mission, is used by the probabilistic engine to simulate potential failures. A random probability generator is used in the engine to determine the end state of the current mission. If the mission is successful, the additional material delivered to a site on the lunar surface and the amount of material consumed is tracked. In this manner a running inventory of surface deliverables is maintained. If the current mission experiences a failure, then the consequences and resultant delays to the remaining missions are determined, based upon specified contingency operational policy. 
Accounting for these consequences a planned manifest is calculated for all remaining missions, including requirements, capacities, loadings, planned cost, crew time, and risk.

The next flight in the scenario is then evaluated and the simulation is repeated until all successive flights have been characterized. The current flight and all the flights that follow are therefore influenced by the events that have occurred cumulatively on all previous flights. After all the flights in a scenario have been simulated, the overall scenario performance for that case is evaluated. The total amount of science conducted, the extensibility objectives met, the additional costs incurred, and crew risk are determined.

\section{B. Collection of Probabilistic Data}

The process of mission-by-mission simulation is repeated for each case that is evaluated in the probabilistic model and a number of cases are generated for each scenario. Each individual case therefore simulates a single possible scenario outcome. Performance data is collected for each case, including surface duration, science mass delivered, crew time available for science, objective satisfaction, mission safety, and total cost. This data is then integrated into probabilistic distributions for expected scenario results. These distributions show the likelihood of achieving different levels of scenario performance based on the current reliability, control policies, and uncertainties within the system. The probability distributions can be compared to the nominal scenario performance, as predicted in deterministic scenario analysis, to evaluate the robustness of the given scenario. Figure 4 shows an example cumulative distribution function (CDF) for total crew days on surface.

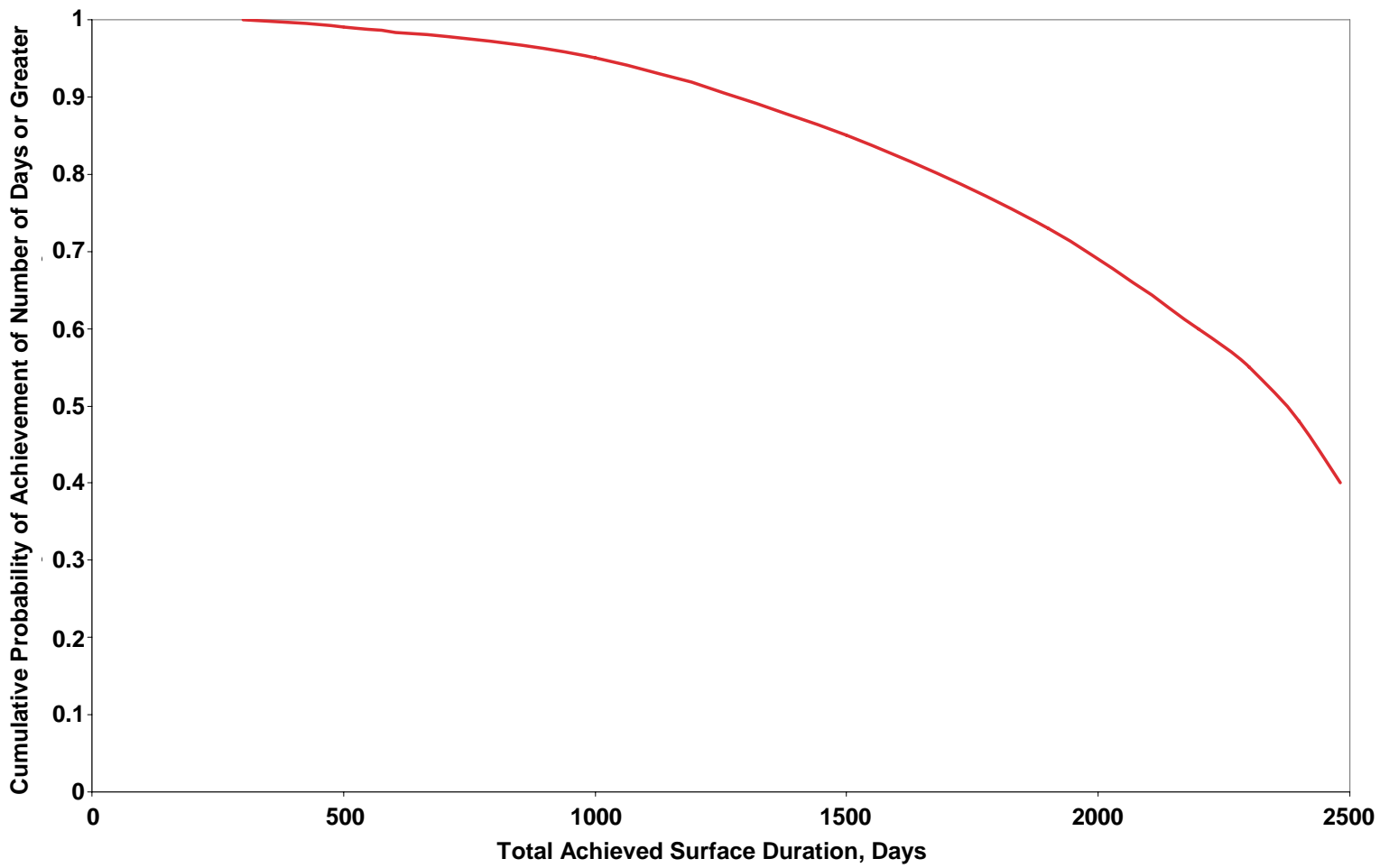

Figure 4. Example Cumulative Distribution Function for Total Crew Days on Surface.

In this example, the nominal planned total surface duration from the deterministic analysis was 2481 days. If every mission had proceeded as planned, this would be the total achieved duration. The results of the probabilistic analysis show that this occurred in only about $40 \%$ of the cases evaluated. The chart also shows that all cases resulted in at least 330 total surface days. The expected value, averaged across all runs, is 1481 surface days.

While the expected value is a key result of the probabilistic analysis, the shape of CDF is also critical for evaluating the robustness of a scenario. The expected value indicates the probabilistic break-even point for performance. There is a $50 \%$ chance that the actual number of surface days achieved will be greater than or equal to this number and a $50 \%$ chance that the actual number of days will be less than this. The shape of the CDF shows how rapidly the expected performance drops off. Figure 5 adds an alternate CDF to the one pictured in Figure 4. 


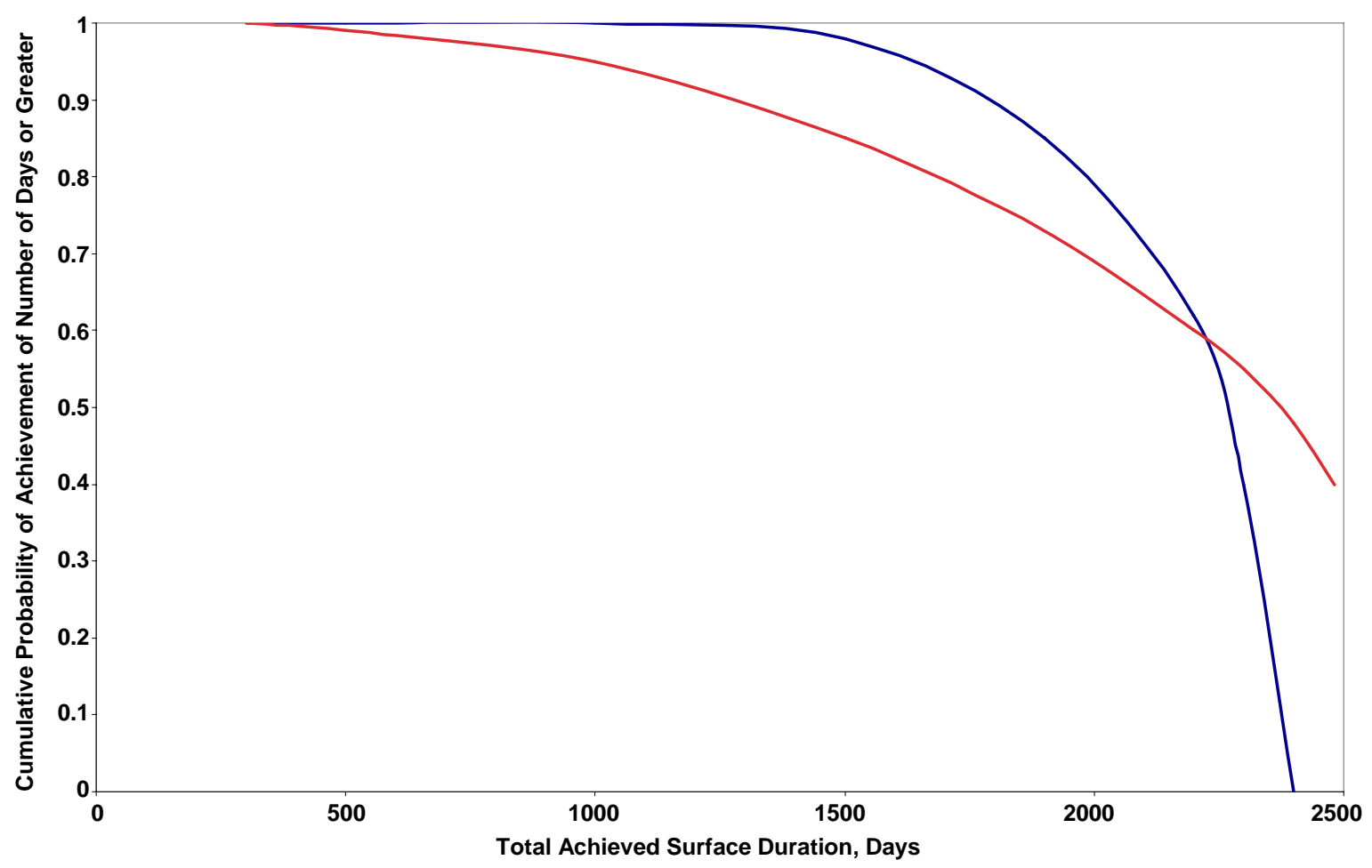

Figure 5. Comparison of Two Example Cumulative Distribution Functions for Total Crew Days on Surface.

In the initial CDF, shown in red, the expected number of surface days drops gradually across the probability range. There is a reasonable probability of achieving the nominal or near-nominal number of days. However, there is also a significant probability of achieving less than $50 \%$ of the planned number of days. In the alternate curve, shown in blue, there is zero probability of achieving the planned number of days. However, the there is also no probability of achieving less than $50 \%$ of the planned number of days. While both of the options may result in a similar expected value, the nature of these results is very different. The red curve is more robust in protecting against high probability failures and has a significant probability of achieving the planned value. However, it is not as robust against less likely failures that have greater impacts. The blue curve is more robust against these high impact failures but less robust against the more likely failures. However, both of these curves have the same expected value for the total number of days achieved.

The total number of individual cases to be run is determined by monitoring the convergence of key variables. Results from all the completed runs are integrated continuously as the model generates them. The expected value of the key parameters, integrated across the cases, is calculated. Initially, the expected value for the parameters will vary significantly as additional runs are completed. However, as more and more runs are completed, the expected values will regress toward the mean. Once the variance in expected results all drop below a certain pre-set level, the model stops generating additional cases. This methodology ensures that the number of runs developed encompasses a broad enough portion of possible real-world cases.

\section{Use of Probabilistic Results}

Results from probabilistic analysis provide decision-makers with additional data regarding the expected productivity of a scenario, the robustness of the scenario, and the effectiveness of contingency planning and policy. This data then allows those decision-makers to make design decisions that are likely to result in acceptable scenario performance, despite the adverse events that may occur.

Decision-making using probabilistic data often requires establishing a risk posture that quantifies the level of acceptable risk. As described above, lunar exploration scenarios that provide a high level of expected performance across the range of possible probabilistic outcomes are identified as being more robust against failures. That is, they are relatively insensitive to the real-world events that disrupt planned behavior. A scenario that exhibits a gradual drop-off in expected performance, as represented in the red curve of Figure 5, will be more robust against the more 
expected failures and will provide a higher probability of achieving near-nominal results. However, such a scenario will also be more vulnerable to major failures and will have a higher probability of achieving very poor performance. If a decision-maker is more willing to accept risk in order to maximize the chances of achieving at or near nominal performance, he or she may gravitate towards this type of scenario, gambling, in effect, that the highlevel of performance can be achieved. If they are less willing to accept risk, and want to ensure that some lesser, still acceptable, level of performance is guaranteed, they might gravitate towards the other option.

Aside from the evaluation of scenario robustness, probabilistic analysis also allows decision-makers to make design decisions regarding element reliability and contingency planning. Scenarios may be developed that provide additional robustness against adverse events through improved contingency policy, better ensuring a high level of expected exploration scenario performance. Typically, however, in order to improve the expected performance under probabilistic conditions, it is necessary to sacrifice some level of nominal performance. Nominal performance is typically traded for increased robustness through increased redundancy, contingency deliveries, schedule margin, or other mitigation techniques.

Probabilistic analysis allows mitigation techniques to be optimized and can demonstrate the ultimate values of these measures to decision-makers, who otherwise tend to focus on nominal performance measures. This additional insight into mitigation of critical failures and the implications for the planned exploration scenario and its associated logistics necessitate the inclusion of probabilistic analysis when defining a scenario.

\section{Conclusions}

Both types of exploration scenario analysis described in this paper are not independent approaches. Rather, they are designed to work together and have been integrated to narrow the scenario trade space in a rigorous but efficient manner. The less computationally and time-intensive deterministic analysis can be used to evaluate a large number of scenario options and to select those that are good candidates for implementation. Probabilistic analysis can then be used to delve deeper into the performance of these high-potential scenarios, evaluating expected performance and robustness. This deeper level of analysis allows decision-makers to optimize the scenario selection and develop contingency planning, ensuring that the final implemented scenario will provide as much value to NASA and its stakeholders as possible.

\section{References}

\footnotetext{
${ }^{1}$ Cirillo, W., Earle, K., Goodliff, K., Reeves, J.D., Andraschko, M., Merrill, R. G., Stromgren, C., "Strategic Analysis Overview,” AIAA Space 2008 Conference \& Exposition, San Diego, California, 2008 (to be published).

${ }^{2}$ Andraschko, M., Merrill, R. G., Earle, K., “Logistics Modeling for Lunar Exploration Systems,” AIAA Space 2008 Conference \& Exposition, San Diego, California, 2008 (to be published).
} 